\title{
COVID-19 outcomes in people with diabetes in Wales: a secondary analysis of the ABCD audit
}

\author{
DAVID M WILLIAMS, ${ }^{1}$ JIM DAVIES, ${ }^{2,3}$ BENJAMIN C T FIELD, ${ }^{4,5}$ RAJIV GANDHI, ${ }^{6}$ SOPHIE HARRIS, ${ }^{7}$ \\ KAMLESH KHUNTI, ${ }^{8}$ DINESH NAGI, ${ }^{9}$ PARTH NARENDRAN, ${ }^{10,11}$ RUSTAM REA, ${ }^{2,12}$ YUE RUAN, ${ }^{2,12}$ \\ ROBERT EJ RYDER, ${ }^{13}$ KINGA A VÁRNAI, ${ }^{2,14}$ SARAH H WILD, ${ }^{15}$ EMMA G WILMOT, ${ }^{16,17}$ \\ THINZAR MIN, ${ }^{1,18,19}$ JULIA PLATTS, ${ }^{20}$ RICHARD CHUDLEIGH, ${ }^{1}$ JEFFREY W STEPHENS, ${ }^{19,21}$ SAM RICE ${ }^{19,22}$
}

\begin{abstract}
Background: People with diabetes and coronavirus disease 2019 (COVID-19) have a significantly greater risk of death and/or intensive care unit (ICU) admission. The Association of British Clinical Diabetologists ( $A B C D$ ) recently audited out-
\end{abstract}

Department of Diabetes \& Endocrinology, Singleton Hospital, Swansea Bay University Health Board, Swansea, UK

Oxford NIHR Biomedical Research Centre, UK

Department of Computer Science, University of Oxford, Oxford, UK

4 Department of Clinical \& Experimental Medicine, Faculty of Health \& Medical Sciences, University of Surrey, Guildford, UK

5 Department of Diabetes \& Endocrinology, Surrey \& Sussex Healthcare NHS Trust, Redhill, Surrey, UK

6 Department of Diabetes \& Endocrinology, Sheffield Teaching Hospitals NHS Foundation Trust, UK

Diabetes and Endocrinology Department, King's College Hospital, UK

8 University Hospitals of Leicester NHS Trust, Diabetes Research Centre, Leicester General Hospital, Leicester, UK

9 Mid Yorkshire Hospitals NHS Trust, Pinderfields Hospital, Wakefield, UK

${ }_{10}$ Medical and Dental Sciences, University of Birmingham, Birmingham, UK

11 Diabetes Centre, The Queen Elizabeth Hospital, University Hospitals Birmingham NHS Foundation Trust, Birmingham, UK

12 Oxford Centre for Diabetes, Endocrinology and Metabolism, Oxford University Hospitals NHS Foundation Trust, UK

13 Sandwell and West Birmingham Hospitals NHS Trust, Birmingham, UK

${ }_{14}$ Oxford University Hospitals NHS Foundation Trust, UK

15 Usher Institute, University of Edinburgh, Edinburgh, UK

${ }_{16}$ Diabetes Department, University Hospitals of Derby and Burton NHS FT, Derby, UK

17 University of Nottingham, Nottingham, UK

18 Department of Diabetes, Neath Port Talbot Hospital, Swansea Bay University Health Board, Neath, UK

19 Diabetes Research Group, Swansea University Medical School, Swansea University, Swansea, UK

20 Diabetes Centre, University Hospital Llandough, Cardiff and Vale University Health Board, Cardiff, UK

21 Department of Diabetes \& Endocrinology, Morriston Hospital, Swansea Bay University Health Board, Swansea, UK

22 Diabetes Centre, Prince Philip Hospital, Hywel Dda University Health Board, Llanelli, UK

Address for correspondence: Dr David M Williams

Department of Diabetes \& Endocrinology, Singleton Hospital, Swansea Bay University Health Board, Swansea, SA2 8QA, UK

E-mail: david.williams@doctors.org.uk

https://doi.org/10.15277/bjd.2021.319 comes for people hospitalised in the UK with diabetes and COVID-19.

Methods: The ABCD COVID-19 and diabetes audit was a retrospective audit of patients admitted to UK hospitals with diabetes and COVID-19 between March and December 2020. Data related to patients admitted in Wales were compared with patients admitted in England and Scotland.

Results: In Wales, 40/82 (48.7\%) patients with diabetes had COVID-19-related mortality compared with 1,149/2,916 $(39.1 \%)$ in the UK group $(p=0.08)$. The Welsh cohort were more likely to be Caucasian, have a higher body mass index and $\mathrm{HbA1c}$, be diagnosed with diabetic retinopathy and prescribed a sodium-glucose co-transporter 2 inhibitor or insulin than those in England and Scotland. Patients admitted to the ICU in Wales were more likely to be male and have type 2 diabetes.

Conclusions: Patients admitted to hospital with diabetes and COVID-19 in Wales had a poorer outcome compared with England and Scotland. This disparity may reflect social inequality, differences in cardiovascular risk factors and/or differences in diabetes medications between hospitalised patients in Wales and the UK.

Br J Diabetes 2021;21:222-227

Key words: COVID-19, diabetes, Wales, United Kingdom

\section{Background}

The extraordinary impact of the severe acute respiratory syndrome coronavirus-2 (SARS-CoV-2) on health systems, global economics and everyday life is unprecedented. At the time of writing, around 187 million cases of coronavirus disease 2019 (COVID-19) have been confirmed worldwide, associated with over 4 million deaths. ${ }^{1}$ Epidemiological studies have found that the risk of death or admission to an intensive care unit (ICU) with COVID-19 is much greater in people with increasing age, significant medical comorbidity (diabetes, cardiovascular disease, renal disease), non-white ethnicity, male gender or social deprivation. ${ }^{2-5}$

Indeed, a third of people who died in the UK during the first wave of COVID-19 in England had underlying diabetes. People with type 1 diabetes (T1D) and type 2 diabetes (T2D) had an odds ratio for in-hospital death of 3.51 (95\% Cl 3.16 to 3.90 ) and 2.03 (95\% 
Cl 1.97 to 2.09), respectively, relative to people without diabetes. ${ }^{6}$ Mortality rates in people with diabetes and COVID-19 in the UK were greater in older people and in those of male gender, non-white ethnicity, socioeconomic deprivation, poorer glycaemic control, obesity and previous renal or cerebrovascular disease and cardiac failure. ${ }^{3}$ Several biological interactions have been suggested to explain the excess risk of poor COVID-19 outcomes in people with diabetes. These include increased angiotensin-converting enzyme2 receptor glycosylation, dipeptidyl peptidase-4 (DPP4) receptor expression, the association between diabetes and obesity, cardiovascular and/or renal disease and possibly an interaction with some pharmacotherapies used to treat diabetes. ${ }^{7}$

In view of the increased risk reported in people with diabetes and COVID-19, the Association of British Clinical Diabetologists $(A B C D)$ recently undertook a UK-wide audit of outcomes in people with diabetes and COVID-19. In this paper we aim to determine the relative outcomes and characteristics of the patients in Wales and compare these with the UK results.

\section{Methods}

The $A B C D$ audit primarily aimed to determine the characteristics and outcomes of people with T1D and T2D admitted to hospital with COVID-19. Secondary aims were to determine whether any patient factors such as ethnicity, obesity, medical comorbidity, glycaemic control or diabetes treatments were associated with the observed health outcomes. Data were collected by clinical teams across the UK and submitted centrally using a specific data collection tool registered to each centre. ${ }^{8}$

Patient data were collected retrospectively for demographics, patient outcomes, body mass index (BMI), glycaemic control, renal function, diabetes complications, medical comorbidities and diabetes treatments across the UK by individual clinical sites. Complete data were collected for 3,413 inpatients admitted to UK hospitals between March and December 2020.8 Data for patients in Wales were extracted from the complete dataset and compared with data from patients in England and Scotland.

\section{Ethical approval}

The audit was registered with Oxford University Hospitals NHS Foundation Trust (OUH) and a data protection impact assessment carried out by the steering group was reviewed by the OUH Caldicott Guardian for use in England and Wales, and by the Public Benefit and Privacy Panel for use in Scotland (reference 2021-0111). ${ }^{8}$ Data collected as part of this audit included routinely collected clinical data only, communicated in pseudonymised form via the secure NHS network. Therefore, this audit did not require research ethics committee approval. ${ }^{9}$

\section{Outcomes}

The primary outcome of this analysis was to determine mortality and ICU admission outcomes of patients admitted to hospitals in Wales with COVID-19 and diabetes. Secondary outcomes were to determine the characteristics of patients admitted to hospitals in Wales, clinical features associated with greater risk of ICU admission and to compare outcomes and clinical characteristics of patients ad- mitted with COVID-19 and diabetes in Wales with the rest of the UK.

\section{Statistical analysis}

Continuous variables are presented as mean (SD) and statistical significance was determined using a paired t-test. Categorical data are presented as the absolute number (\%) and statistical significance determined using a $\mathrm{X}^{2}$ test. Statistical significance was considered at $p<0.05$.

\section{Results}

Clinical characteristics and outcomes of Welsh population Clinical characteristics of the 104 patients with diabetes and COVID19 in Wales are presented in Table 1. Some data are missing and the data available are presented. Patients had a mean age of 72 years and $55.7 \%$ were male with a mean BMl of $33.5 \mathrm{~kg} / \mathrm{m}^{2}$ and mean $\mathrm{HbA}_{1 \mathrm{c}}$ of $67 \mathrm{mmol} / \mathrm{mol}$ (8.3\%). Table 2 summarises the preexisting diabetes-associated complications and diabetes pharmacotherapy prescribed for these patients. At presentation to hospital services, $5.8 \%$ of patients admitted in Wales were in diabetic ketoacidosis (DKA).

During admission to hospital with COVID-19, 45/104 (43.2\%) patients in Wales died and/or were admitted to the adult intensive care unit (AICU) and 40/82 (48.7\%) of the patients died (missing data for 22 patients). Greater mortality was observed with increasing age in these patients: $<55$ years, 3/11 (27.3\%); 55-74 years, 15/34 (44.1\%) and $\geq 75$ years, 22/37 (59.5\%) patients died. In the Welsh cohort, death and/or admission to AICU were significantly more likely in males and in patients with T2D rather than T1D, as shown in Table 3. There was no statistically significant association between death and/or admission to AICU with ethnicity, age, BMI, admission blood glucose, HbA1c, creatinine, medical comorbidity or diabetes pharmacotherapy in the Welsh cohort.

\section{Comparing Welsh and UK outcomes and population characteristics}

As shown in Table 1, people in Wales admitted with diabetes and COVID-19 had a non-significant trend for increased mortality compared with those admitted in the rest of the UK (40/82 (48.7\%) vs $1,149 / 2,916(39.1 \%), p=0.08)$ and a similar rate of death and/or admission to AICU. Patients admitted in Wales were significantly more likely to be Caucasian, have a greater BMI or HbA1c and had a comparable rate of medical comorbidities compared with patients admitted across the rest of the UK.

Patients with COVID-19 and diabetes admitted to hospital in Wales were significantly more likely to have diabetic retinopathy and be prescribed a sodium-glucose co-transporter 2 (SGLT-2) inhibitor or insulin than patients admitted across the rest of the UK. There were no significant differences in the rates of other diabetes complications including DKA or medication prescriptions between the groups (Table 2).

\section{Discussion}

In this secondary analysis of the ABCD audit we aimed to determine the outcomes of the 104 patients admitted with diabetes and 
Table 1 Comparison of the major outcomes of death or AICU admission, patient demographics and comorbidity between patients with diabetes and COVID-19 in Wales and the UK

\begin{tabular}{|c|c|c|c|c|}
\hline Clinical features & Wales $(n=104)$ & UK $(n=3309)$ & Hazard ratio & $P$ value \\
\hline Death and/or AICU & $45 / 104(43.2 \%)$ & $1319 / 2976(44.3 \%)$ & 0.98 & 0.83 \\
\hline Mean (SD) age (years) & $72(13)$ & $72(14)$ & - & 1.00 \\
\hline Ethnicity & $\begin{array}{l}\text { White }(71 / 80,88.8 \%) \\
\text { Asian }(1 / 80,1.2 \%) \\
\text { Black }(2 / 80,2.5 \%) \\
\text { Other }(6 / 80,7.5 \%)\end{array}$ & $\begin{array}{l}\text { White }(1839 / 2836,64.8 \%) \\
\text { Asian }(472 / 2836,16.6 \%) \\
\text { Black }(218 / 2836,7.7 \%) \\
\text { Other }(317 / 2836,11.1 \%)\end{array}$ & - & $\begin{array}{l}<0.01 \\
<0.01 \\
0.08 \\
0.30\end{array}$ \\
\hline $\mathrm{BMI}\left(\mathrm{kg} / \mathrm{m}^{2}\right)$ & $33.5(7.7)$ & $29.3(7.1)$ & - & $<0.01$ \\
\hline Admission blood glucose (mmol/L) & $11.8(7.4)$ & $11.1(6.8)$ & - & 0.34 \\
\hline $\mathrm{HbA}_{1 \mathrm{c}}(\mathrm{mmol} / \mathrm{mol})$ & $67(24)$ & $57(26)$ & - & $<0.01$ \\
\hline Creatinine $(\mu \mathrm{mol} / \mathrm{L})$ & $144(110)$ & $153(162)$ & - & 0.42 \\
\hline Hypertension & $70 / 96(72.9 \%)$ & $2045 / 2943(69.5 \%)$ & 1.05 & 0.47 \\
\hline Malignant neoplasm & $14 / 94(14.9 \%)$ & $436 / 2819(15.5 \%)$ & 0.88 & 0.88 \\
\hline Smoker & $6 / 78(7.7 \%)$ & $123 / 1575(7.8 \%)$ & 0.98 & 0.97 \\
\hline
\end{tabular}

Table 2 Comparison of the rates of diabetes complications and diabetes pharmacotherapy prescription between patients with COVID-19 and diabetes in Wales and the UK

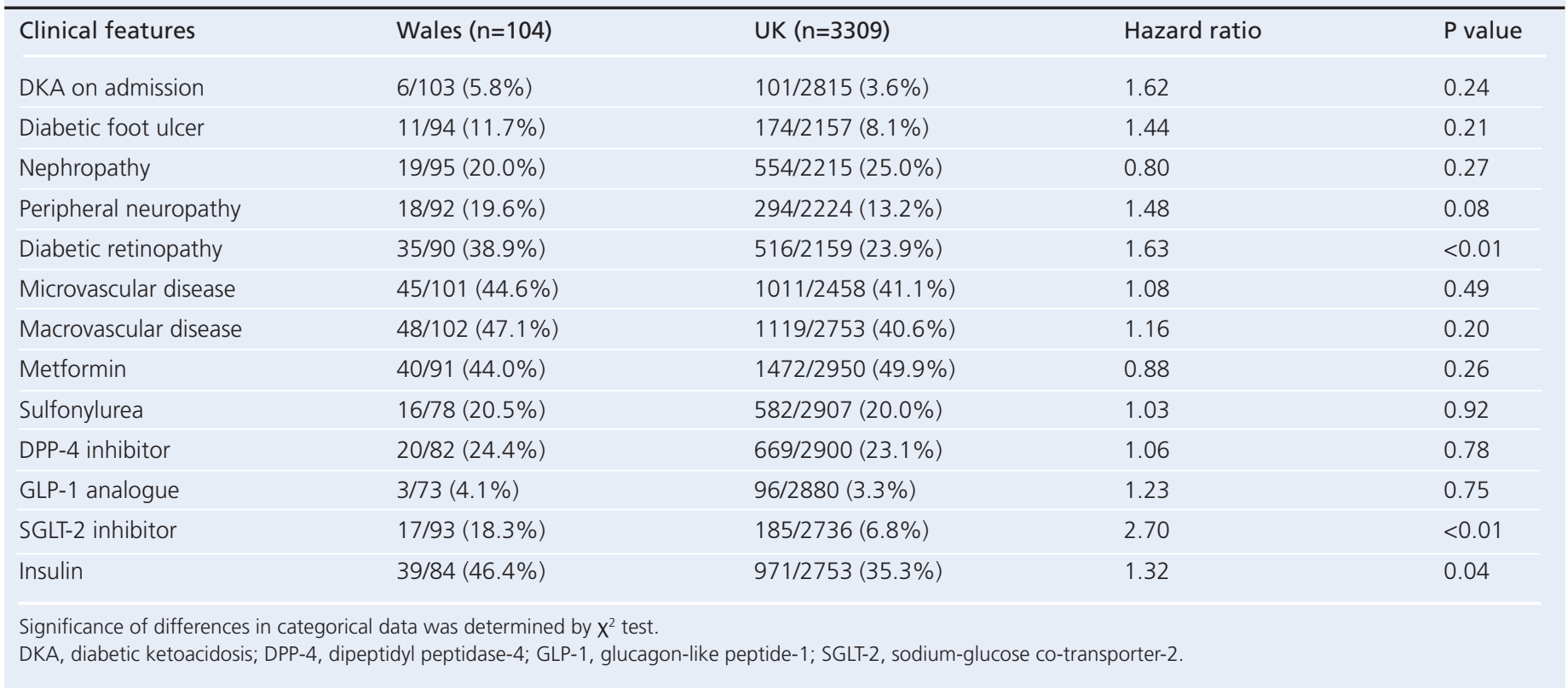

COVID-19 in Wales and compare these outcomes to those in the rest of the UK. We observed that $48.7 \%$ of the patients with diabetes and COVID-19 admitted in Wales died, around 10\% more than that observed in England and Scotland. We also observed that patients admitted in Wales were more likely to be Caucasian, have a greater $\mathrm{BMI}, \mathrm{HbA}_{1 \mathrm{c}}$ and have co-morbid retinopathy compared with the rest of the UK. Of the patients in Wales who died and/or were admitted to AICU, there was a significantly greater male prevalence and number of patients with T2D compared with T1D. However, we did not observe any significant difference in anthropometric measures, medical comorbidities or diabetes-related complications associated with an increased risk of death and/or AICU admission in the Wales cohort. Similarly, we did not find an association between any diabetes treatments with death and/or AICU admission. 
Table 3 Comparison of demographic data, diabetes complications and diabetes treatment between patients with diabetes and COVID-19 who died and/or were admitted to the AICU against patients who survived COVID-19 and were not admitted to the AICU in Wales

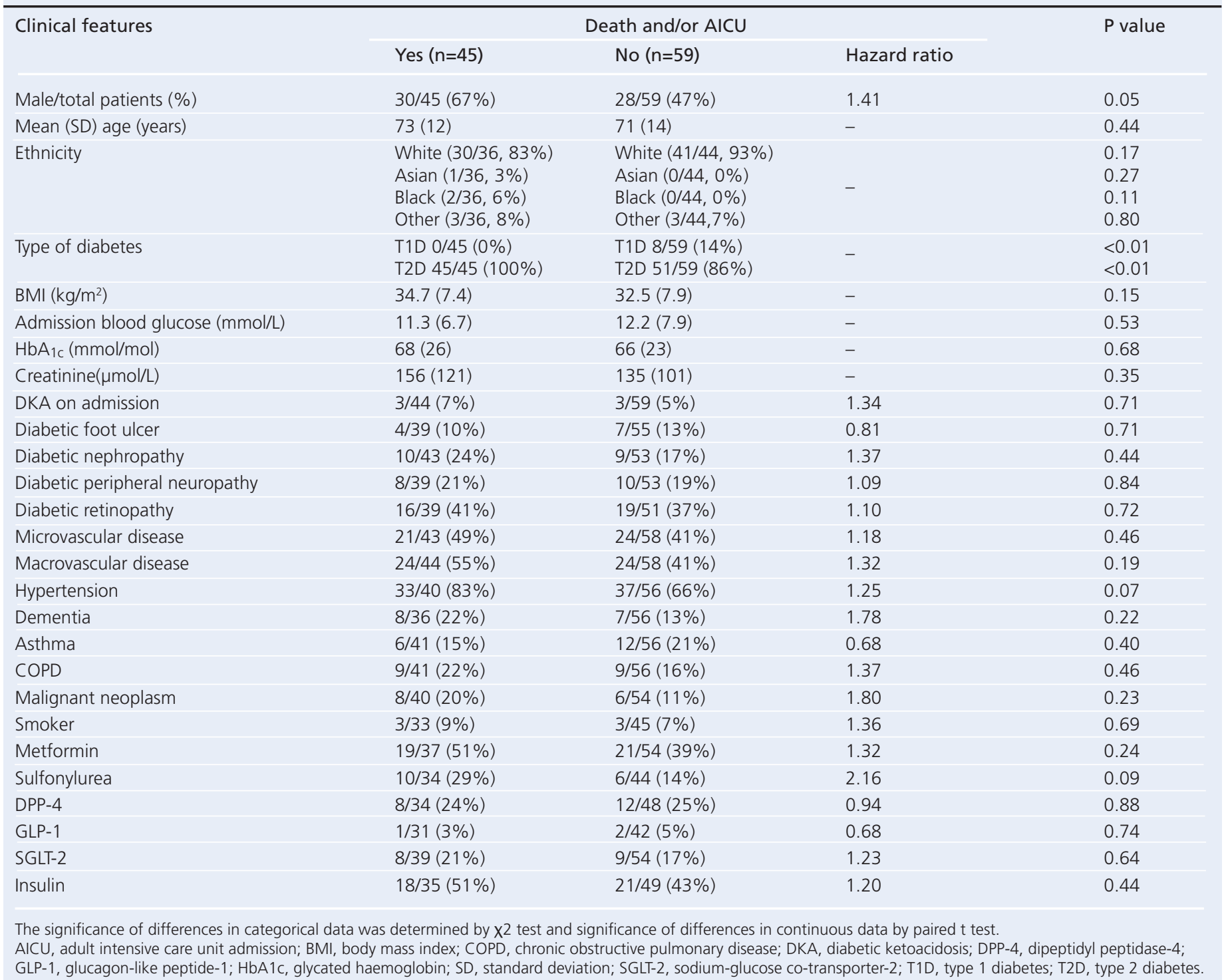

Given that patients in the Welsh cohort were significantly more likely to be Caucasian and trended to a lower proportion of male patients, it may seem surprising that these patients demonstrated a higher mortality considering previous UK COVID-19 epidemiological observations. ${ }^{3}$ There are several possibilities which may explain the poorer outcomes observed in the Welsh population. The first is that of social deprivation, and its impact upon health behaviours and outcomes is well known. ${ }^{10}$ Indeed, greater social deprivation has been previously associated with higher morbidity and mortality in patients with COVID-19 in populations in both Wales and the UK generally. ${ }^{2,5}$ In one study $42.3 \%$ of patients admitted to the ICU with COVID-19 resided in geographical areas representing the $20 \%$ most deprived in Wales and were therefore greatly over-represented in the ICU cohort. ${ }^{2}$ Whilst the relative social deprivation of our cohort compared with the rest of the UK is unknown from the limited data related to social deprivation available, previous studies have observed that people in Wales are significantly more deprived than those in England or Scotland. ${ }^{11}$

Patients in the Welsh cohort were observed to demonstrate a greater prevalence of risk factors associated with poorer health outcomes such as greater $\mathrm{HbA}_{1 \mathrm{c}}$ and $\mathrm{BMI}$. Indeed, in the national diabetes audit 2018-2019, patients with either T1D or T2D in Wales were less likely to attain a target $\mathrm{HbA}_{1 \mathrm{c}}<48 \mathrm{mmol} / \mathrm{mol}(6.5 \%),<58$ $\mathrm{mmol} / \mathrm{mol}(7.5 \%)$ or $<86 \mathrm{mmol} / \mathrm{mol}(10.0 \%)$ than patients in England. ${ }^{12}$ Moreover, the obesity rate in adults in Wales is increasing and the rate of obesity in 15-year-olds is one of the highest in Europe. ${ }^{13,14}$ This may reflect poorer health behaviours associated with greater social deprivation in the Welsh cohort compared with that of the rest of the UK. Irrespective of their relative social deprivation, these greater risk factors will have contributed to the greater mor- 
tality observed in the Welsh group compared with the rest of the UK. Furthermore, in the Welsh cohort there was a trend to greater $\mathrm{BMI}$ in those who died and/or were admitted to AICU compared with those who were not (BMl 34.7 vs. $32.5 \mathrm{~kg} / \mathrm{m}^{2}, \mathrm{p}=0.15$ ). The influence of obesity on patient outcomes is stressed by epidemiological studies observing greater COVID-19 mortality in patients with increasing BMI. ${ }^{15,16}$

Multiple factors may explain the greater risk of death in people with obesity, including a pro-inflammatory state, insulin resistance, ACE2 receptor expression in adipocytes and greater difficulties associated with intubation. Nevertheless, this association is confounded by the complex inter-relationship between obesity, medical comorbidity and social deprivation. ${ }^{10} \mathrm{~A}$ further possibility is that differences in the medications prescribed for diabetes between the two groups affected patient outcomes. There had been major concerns at the start of the pandemic around the prescription of diabetes medications such as SGLT-2 inhibitors for people with diabetes during the pandemic, due to a potentially greater risk of diabetic ketoacidosis (DKA) associated with their use which may be exacerbated by SARS-CoV-2. ${ }^{17}$ Patients admitted in Wales were significantly more likely to be prescribed SGLT-2 inhibitors or insulin compared with patients admitted in the rest of the UK. Similarly, patients admitted to hospitals in Wales had a numerically - though not statistically significant - greater risk of presentation with DKA compared with the rest of the UK ( $5.8 \%$ vs $3.6 \%, p=0.24)$. However, as shown in Table 3, there was no statistically significant difference in the prevalence of SGLT-2 inhibitor prescription between patients who died and/or those admitted to AICU in Wales. The increased rate of insulin prescription in patients admitted to hospital in Wales compared with the UK likely reflects the poorer glycaemic control in this cohort and therefore their more advanced pharmacotherapy for diabetes. Nevertheless, previous analyses have observed that insulin prescription is associated with a greater risk of COVID-19-related death in people with $T 2 D,{ }^{18}$ although this is likely an association observed in a cohort with more complicated diabetes and/or comorbidity rather than a causative relationship.

To mitigate the difference in mortality, efforts to address modifiable risk factors for poorer outcomes in this cohort are essential. To achieve this, improving the national attainment of the NICE recommended eight care processes and three treatment targets is important. Indeed, Wales had a poorer performance than England with respect to completion of these targets in the national diabetes audit 2017-18. Moreover, patients had less time on average with specialist diabetes nurses, specialist dieticians and podiatrists in Wales compared with England. ${ }^{19}$ Addressing such inequalities between the delivery of diabetes care in Wales and the rest of the UK will likely reduce the prevalence of the risk factors presented in this analysis. This may lessen the observed difference in outcomes associated with severe illness such as COVID-19 in the future.

\section{Limitations}

There are some important limitations to this analysis. Importantly, the sample number of the patients admitted to hospitals in Wales $(n=104)$ was relatively much smaller than the total number included in the UK (England and Scotland) dataset $(n=3,309)$. Given the ret-

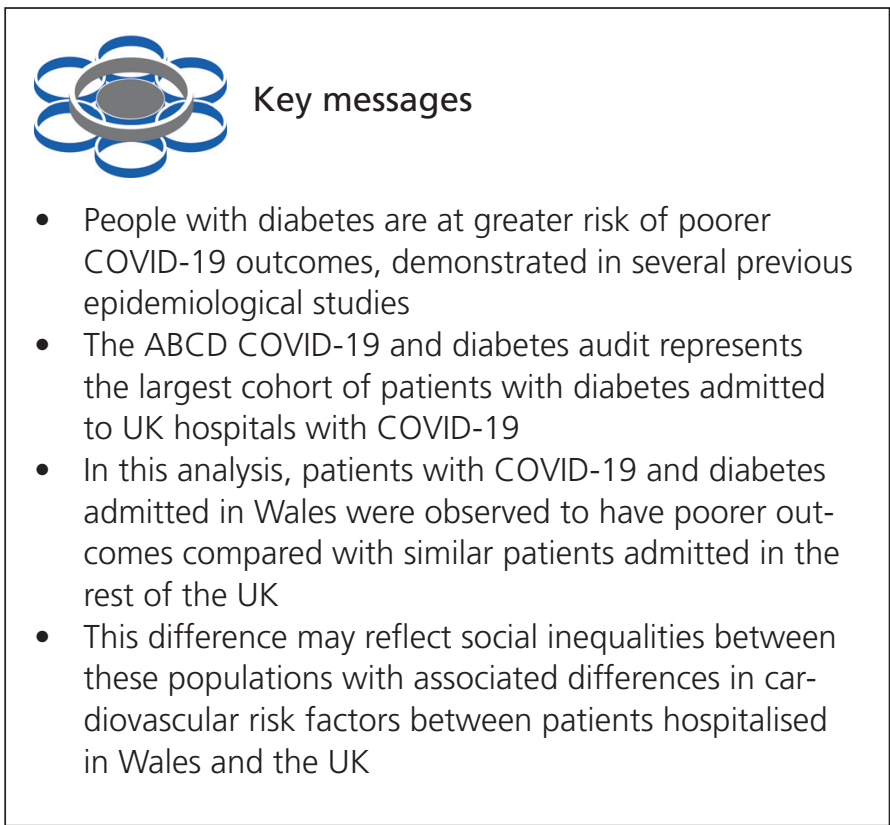

rospective nature of the data collected, these data are disposed to the usual biases affecting this type of study and there may be incomplete case ascertainment and reporting of patients with diabetes and COVID-19. Particularly within the Wales dataset, there were missing data with respect to death outcomes in 22 patients (21.1\% of the patients in Wales included in this analysis). Unfortunately, there was insufficient data related to social deprivation to allow comparison between patients in Wales and the rest of the UK including multivariate analysis for independent risk factors for death and/or AICU admission. These data are also limited by the lack of a control group of people with COVID-19 without diabetes.

\section{Conclusion}

The impact of COVID-19 in the UK and worldwide has been unprecedented, and the impact felt by people with diabetes has been even greater. This audit undertaken by the $A B C D$ represents the largest cohort of people with diabetes admitted to NHS hospitals in the UK with COVID-19. This analysis highlights important differences in the clinical outcomes and characteristics of patients with diabetes in Wales compared with rest of the UK. These differences may result from several possibilities including social deprivation, medication prescription and control of the underlying cardiovascular risk factors observed in these populations.

Acknowledgements: We would like to thank the clinical teams who collected the data for this audit and the ABCD for providing the dataset used to produce this manuscript.

Authorship: All named authors meet the International Committee of Medical Journal Editors (ICMJE) criteria for authorship of this article, take responsibility for the integrity of the work and have given their approval for this version to be published.

Conflict of interest: KK is supported by the National Institute for Health Research (NIHR) Applied Research Collaboration East Midlands (ARC EM) and the NIHR Leicester Biomedical Research Centre (BRC); grants/contracts: Boehringer Ingelheim, AstraZeneca, Novar- 
tis, Novo Nordisk, Sanofi-Aventis, Lilly, Merck Sharp \& Dohme; honoraria for lectures: Amgen, AstraZeneca, Bayer, NAPP, Lilly, MSD, Novartis, Novo Nordisk, Roche, Berlin-Chemie AG/Menarini Group, Sanofi-Aventis, Servier, Boehringer Ingelheim. YR is supported by a Novo Nordisk Post-Doctoral Fellowship run in partnership with the University of Oxford. RR is supported by the National Institute for Health Research (NIHR) Oxford Biomedical Research Centre. Data Monitoring Committee for Impact of Semaglutide in Amyloid Positivity funded by NovoNordisk (no payment). RC: payment or honoraria for lectures from Novo Nordisk, MSD, Boehringer Ingelheim, AstraZeneca; support for attending meetings from Boehringer Ingelheim and AstraZeneca. BF: payment or honoraria for lectures from Abbott, AstraZeneca, Boehringer Ingelheim, Eli Lilly, Gilead, Janssen, Medtronic, MSD, Napp, Novo Nordisk, Sanofi; support for attending meetings from Novo Nordisk and Sanofi; participation on Data Safety Monitoring Board/Advisory Board: Abbott, Boehringer Ingelheim, Novo Nordisk. TM: payment or honoraria for lectures from AstraZeneca, Boehringer Ingelheim. JS: payment or honoraria from lectures from Eli Lilly, Novo Nordisk, Boehringer Ingelheim, AstraZeneca; attended American Diabetes Association with support from Boeringer Ingelheim, 2019. REJR has received speaker fees, and/or consultancy fees and/or educational sponsorships from AstraZeneca, BioQuest, GI Dynamics, Janssen and Novo Nordisk.

Funding: This audit was part funded by a grant from the all-Wales Diabetes Implementation group. YR's salary was funded by Novo Nordisk Postdoctoral Fellowship run in partnership with the University of Oxford. RR is partly funded by the National Institute for Health Research (NIHR) Oxford Biomedical Research Centre (BRC).

\section{References}

1. World Health Organisation. WHO Coronavirus (COVID-19) Dashboard. Available at: https://covid19.who.int/ [Accessed 13 July 2021].

2. Baumer T, Phillips E, Dhadda A, Szakmany T. Epidemiology of the first wave of COVID-19 ICU admissions in South Wales: the interplay between ethnicity and deprivation. Front Med (Lausanne) 2020;7:569714. https://doi.org/10.3389/fmed.2020.569714

3. Holman N, Knighton P, Kar P, et al. Risk factors for COVID-19-related mortality in people with type 1 and type 2 diabetes in England: a population-based cohort study. Lancet Diabetes Endocrinol 2020;8(10):82333. https://doi.org/10.1016/S2213-8587(20)30271-0

4. Booth $A$, Reed $A B$, Ponzo $S$, et al. Population risk factors for severe disease and mortality in COVID-19: a global systematic review and metaanalysis. PLoS One 2021;16(3):e0247461. https://doi.org/10.1371/ journal.pone. 0247461

5. Woodward M, Peters SAE, Harris K. Social deprivation as a risk factor for COVID-19 mortality among women and men in the UK Biobank: nature of risk and context suggests that social interventions are essential to mitigate the effects of future pandemics. J Epidemiol Community Health 2021;75(11):1050-5. http://dx. doi.org/10.1136/jech-2020215810
6. Barron E, Bakhai C, Kar P, et al. Associations of type 1 and type 2 diabetes with COVID-19-related mortality in England: a whole-population study. Lancet Diabetes Endocrinol 2020;8(10):813-22. https://doi.org/ 10.1016/S2213-8587(20)30272-2

7. Williams DM, Nawaz A, Evans M. Diabetes and novel coronavirus infection: implications for treatment. Diabetes Ther 2020;11:1915-24. https://doi.org/10.1007/s13300-020-00858-2

8. Nagi D, Ryder REJ, Ruan Y, et al. An audit of people admitted to hospital with diabetes and coronavirus (SARS-CoV-2): data collection methods. The Association of British Clinical Diabetologists (ABCD) Nationwide Audit. $\mathrm{Br} J$ Diabetes 2021;21(1):96-9. https://doi.org/10.15277/ bjd.2021.299.

9. Oxford University Hospitals. Is my project research? Available from: https://www.ouh.nhs.uk/researchers/planning/is-it-research/ [Accessed 13 July 2021]

10. Foster HME, Celis-Morales CA, Nicholl Bl, et al. The effect of socioeconomic deprivation on the association between an extended measurement of unhealthy lifestyle factors and health outcomes: a prospective analysis of the UK Biobank cohort. Lancet Public Health 2018; 3(12):e576-e585. https://doi.org/10.1016/\$2468-2667(18)30200-7

11. Abel GA, Barclay ME, Payne RA. Adjusted indices of multiple deprivation to enable comparisons within and between constituent countries of the UK including an illustration using mortality rates. BMJ Open 2016; 6:e012750. http://dx.doi.org/10.1136/bmjopen-2016-012750

12. NHS Digital. 2020. National Diabetes Audit - Report 1. Care Processes and Treatment Targets 2018-19, Full Report. Available at: https://digital.nhs.uk/data-and-information/publications/statistical/national-diabetes-audit/report-1-care-processes-and-treatment-targets-2018-19-fullreport [Accessed 13 July 2021].

13. Public Health Wales. 2018. The case for action on obesity in Wales. Available at: https://phw.nhs.wales/topics/overweight-and-obesity/the-casefor-action-on-obesity-in-wales/ [Accessed 13 July 2021].

14. Welsh Government. 2019. Obesity Pathway. Available at: https://gov.wales/sites/default/files/publications/2019-06/obesity-pathway.pdf\#: :text=In\%20Wales\%2C\%20as\%20in\%20many\%20other\% 20 countries $\% 2$ C \% 20the, $72 \% 25 \% 20$ and $\% 2062 \% 25 \% 2$ C \% 20respectively\%20among \%2045-64\%20year\%20olds [Accessed 13 July 2021].

15. Gao M, Piernas C, Astbury NM, et al. Associations between body-mass index and COVID-19 severity in 6.9 million people in England: a prospective, community-based, cohort study. Lancet Diabetes Endocrinol 2021; 9(6):350-9. https://doi.org/10.1016/S2213-8587(21)00089-9

16. Cao P, Song Y, Zhuang Z, et al. Obesity and COVID-19 in adult patients with diabetes. Diabetes 2021;70(5):1061-9. https://doi.org/10.2337/ db20-0671

17. Scheen AJ. SGLT2 inhibition during the COVID-19 epidemic: friend or foe? Diabetes Metab 2020;46(5):343-4. https://doi.org/10.1016/j.diabet.2020.06.003

18. Khunti K, Knighton P, Zaccardi F, et al. Prescription of glucose-lowering therapies and risk of COVID-19 mortality in people with type 2 diabetes: a nationwide observational study in England. Lancet Diabetes Endocrinol 2021;9(5):293-303. https://doi.org/10.1016/\$2213-8587(21)00050-4

19. Diabetes UK. The state of the nation 2019: a review of diabetes services in Wales. Available at: https://www. diabetes.org.uk/resources-s3/public/202006/SOTN\%20report\%20ENGLISH\%5BW\%5D_0.pdf?WQLn4oGFIPmQi3f_ SQjptcKkAelHTXdn= [Accessed 13 July 2021]. 